\title{
Dark Matter: S0-2's Gravitational Redshift Could Give an Important Clue to Solve the Dark Matter Mystery
}

\author{
Stéphane Le Corre \\ Ecole Polytechnique Fédérale de Lausanne, Lausanne, Switzerland \\ Email: stephane.lecorre@epfl.ch
}

How to cite this paper: Le Corre, S. (2018) Dark Matter: SO-2's Gravitational Redshift Could Give an Important Clue to Solve the Dark Matter Mystery. Open Access Library Journal, 5: e5028.

https://doi.org/10.4236/oalib.1105028

Received: November 5, 2018

Accepted: November 26, 2018

Published: November 29, 2018

Copyright (c) 2018 by author and Open Access Library Inc.

This work is licensed under the Creative Commons Attribution International License (CC BY 4.0).

http://creativecommons.org/licenses/by/4.0/

\begin{abstract}
The star SO-2 at the galactic center was recently at its closest distance to the supermassive black hole (SMBH). It allowed measuring relativistic effects. The observations confirm that the general relativity is the best gravitational theory compared to other alternative theories [1]. But although an excellent agreement with the observations is obtained all along the orbit, a discrepancy in the redshift of SO-2 is measured at its periastron. An excess of around 20\% $(240 \mathrm{~km} / \mathrm{s}$ instead of $200 \mathrm{~km} / \mathrm{s})$ has been observed. This discrepancy has been predicted in the paper [2]. It should come from the second component (gravitic field) of the general relativity generated by SgrA ${ }^{*}$. In general, the expected value of this component of general relativity is negligible. But in the frame of the explanation of dark matter (as the gravitic field of the clusters [3]), the gravitic field of the galaxies should be larger than expected. It is in this theoretical frame (without exotic matter and in agreement with general relativity) that this discrepancy was predicted. Furthermore, its value is in agreement with the expected gravitic field computed in [3]. These results would mean that the gravitic field of large astrophysical structures (galaxies, clusters, ...) would be greater than expected and that the explanation of dark matter by the gravitic field could be a pertinent solution (in agreement with general relativity and without exotic matter). Furthermore, this explanation implies necessarily movements of dwarf satellite galaxies along planes, movements that seem to be more and more likely and allows retrieving experimental relations. This dark matter explanation would then solve several kinds of problems with this specific component of general relativity currently neglected.
\end{abstract}

\section{Subject Areas}

Modern Physics 


\section{Keywords}

Galaxies: Fundamental Parameters, Galaxies: General, Galaxies: Kinematics and Dynamics, Dark Matter

\section{Introduction}

General relativity implies the existence of two gravitational components. In addition to the gravity field, there is a gravitic field just like the magnetic field in electromagnetism. These both components give what is called the gravitomagnetism, obtained from the linearization of the general relativity. This new gravitic field can be measured by its effect, known as Lense-Thirring effect. Several experiments have validated this effect for the Earth gravitic field, NASA's LAGEOS satellites or Gravity Probe B [4]. In [3], a solution is proposed to explain the dark matter, compliant with general relativity and without exotic matter. This explanation leads to the assumption that we are embedded in a relatively uniform gravitic field generated by larger structures than galaxies (likely the clusters). More generally, this solution implies that large structures generate greater gravitic fields (and then Lense-Thirring effect) than expected. In particular the center of galaxies could be a first example of such a phenomenon. Even if the gravitic field of the galaxies' center can't explain the dark matter at the ends of the galaxies [3], it could be locally an important component of the dynamic of the object near the center (sometime of the same order of the gravity field). The goal of this study is to explain how the discrepancy of the measured redshift of SO-2 at the periastron can be explained in the frame of the general relativity (following the computed values obtained to explain dark matter in [3]). Consequently, this observation associated with this explanation would give an important clue for the dark matter explanation of [3].

Let's first demonstrate what general relativity gives for the expressions of the gravitic field; it will allow giving us an expression for the gravitational redshift.

\section{Dark Matter Explained by General Relativity}

\subsection{From General Relativity to Linearized General Relativity}

From general relativity, one deduces the linearized general relativity in the approximation of a quasi-flat Minkowski space $\left(g^{\mu v}=\eta^{\mu v}+h^{\mu \nu} ;\left|h^{\mu \nu}\right| \ll 1\right)$. With the following Lorentz gauge, it gives the following field equations as in [5] (with $\left.\square=\frac{1}{c^{2}} \frac{\partial^{2}}{\partial t^{2}}-\Delta\right)$ :

$$
\partial_{\mu} \bar{h}^{\mu v}=0 ; \quad \square \bar{h}^{\mu v}=-2 \frac{8 \pi G}{c^{4}} T^{\mu v}
$$

with:

$$
\bar{h}^{\mu v}=h^{\mu v}-\frac{1}{2} \eta^{\mu v} h ; h \equiv h_{\sigma}^{\sigma} ; h_{v}^{\mu}=\eta^{\mu \sigma} h_{\sigma v} ;
$$




$$
\bar{h}=-h
$$

The general solution of these equations is:

$$
\bar{h}^{\mu v}(c t, \boldsymbol{x})=-\frac{4 G}{c^{4}} \int \frac{T^{\mu v}(c t-|\boldsymbol{x}-\boldsymbol{y}|, \boldsymbol{y})}{|\boldsymbol{x}-\boldsymbol{y}|} \mathrm{d}^{3} \boldsymbol{y}
$$

In the approximation of a source with low speed, one has:

$$
T^{00}=\rho c^{2} ; T^{0 i}=c \rho u^{i} ; T^{i j}=\rho u^{i} u^{j}
$$

And for a stationary solution, one has:

$$
\bar{h}^{\mu v}(\boldsymbol{x})=-\frac{4 G}{c^{4}} \int \frac{T^{\mu v}(\boldsymbol{y})}{|\boldsymbol{x}-\boldsymbol{y}|} \mathrm{d}^{3} \boldsymbol{y}
$$

At this step, because these approximations lead to an idealization equivalent to electromagnetism, one traditionally defines a scalar potential $\varphi$ and a vector potential $H^{i}$. There are in the literature several definitions as in [6] for the vector potential $H^{i}$. In our study, we are going to define:

$$
\bar{h}^{00}=\frac{4 \varphi}{c^{2}} ; \bar{h}^{0 i}=\frac{4 H^{i}}{c} ; \bar{h}^{i j}=0
$$

with gravitational scalar potential $\varphi$ and gravitational vector potential $H^{i}$ :

$$
\begin{aligned}
& \varphi(\boldsymbol{x}) \equiv-G \int \frac{\rho(\boldsymbol{y})}{|\boldsymbol{x}-\boldsymbol{y}|} \mathrm{d}^{3} \boldsymbol{y} \\
& H^{i}(\boldsymbol{x}) \equiv-\frac{G}{c^{2}} \int \frac{\rho(\boldsymbol{y}) u^{i}(\boldsymbol{y})}{|\boldsymbol{x}-\boldsymbol{y}|} \mathrm{d}^{3} \boldsymbol{y}=-K^{-1} \int \frac{\rho(\boldsymbol{y}) u^{i}(\boldsymbol{y})}{|\boldsymbol{x}-\boldsymbol{y}|} \mathrm{d}^{3} \boldsymbol{y}
\end{aligned}
$$

with $K$ a new constant defined by:

$$
G K=c^{2}
$$

This definition gives $K^{-1} \sim 7.4 \times 10^{-28}$ very small compare to $G$.

The field Equation (1) can be then written (Poisson equations):

$$
\Delta \varphi=4 \pi G \rho ; \Delta H^{i}=\frac{4 \pi G}{c^{2}} \rho u^{i}=4 \pi K^{-1} \rho u^{i}
$$

with the following definitions of $\boldsymbol{g}$ (gravity field) and $\boldsymbol{k}$ (gravitic field), those relations can be obtained from the following equations (also called gravitomagnetism):

$$
\begin{aligned}
& \boldsymbol{g}=-\operatorname{grad} \varphi ; \boldsymbol{k}=\boldsymbol{r o t} \boldsymbol{H} \\
& \operatorname{rot} \boldsymbol{g}=0 ; \operatorname{div} \boldsymbol{k}=0 ; \\
& \operatorname{div} \boldsymbol{g}=-4 \pi G \rho ; \boldsymbol{r o t} \boldsymbol{k}=-4 \pi K^{-1} \boldsymbol{j}_{p}
\end{aligned}
$$

with the Equation (2), one has:

$$
h^{00}=h^{11}=h^{22}=h^{33}=\frac{2 \varphi}{c^{2}} ; h^{0 i}=\frac{4 H^{i}}{c} ; h^{i j}=0
$$

The equations of geodesics in the linear approximation give:

$$
\frac{\mathrm{d}^{2} x^{i}}{\mathrm{~d} t^{2}} \sim-\frac{1}{2} c^{2} \delta^{i j} \partial_{j} h_{00}-c \delta^{i k}\left(\partial_{k} h_{0 j}-\partial_{j} h_{0 k}\right) v^{j}
$$


It then leads to the movement equations:

$$
\frac{\mathrm{d}^{2} \boldsymbol{x}}{\mathrm{d} t^{2}} \sim-\operatorname{grad} \varphi+4 \boldsymbol{v} \wedge(\boldsymbol{r o t} \boldsymbol{H})=\boldsymbol{g}+4 \boldsymbol{v} \wedge \boldsymbol{k}
$$

From relation (11), one deduces the metric in a quasi flat space:

$$
\mathrm{d} s^{2}=\left(1+\frac{2 \varphi}{c^{2}}\right) c^{2} \mathrm{~d} t^{2}+\frac{8 H_{i}}{c} c \mathrm{~d} t \mathrm{~d} x^{i}-\left(1-\frac{2 \varphi}{c^{2}}\right) \sum\left(\mathrm{d} x^{i}\right)^{2}
$$

In the approximation of a quasi-flat Minkowski space, one has:

$$
H_{i} \mathrm{~d} x^{i}=-\delta_{i j} H^{j} \mathrm{~d} x^{i}=-\boldsymbol{H} \cdot \mathrm{d} \boldsymbol{x}
$$

We retrieve the known expression [5] with our definition of $H_{i}$ :

$$
\mathrm{d} s^{2}=\left(1+\frac{2 \varphi}{c^{2}}\right) c^{2} \mathrm{~d} t^{2}-\frac{8 \boldsymbol{H} \cdot \mathrm{d} \boldsymbol{x}}{c} c \mathrm{~d} t-\left(1-\frac{2 \varphi}{c^{2}}\right) \sum\left(\mathrm{d} x^{i}\right)^{2}
$$

Remark: The interest of our notation (compare to the traditional notation of gravitomagnetism) is that the field equations are strictly equivalent to Maxwell idealization (in particular the speed of the gravitational wave obtained from these equations is the light celerity). Only the movement equations are different with the factor " 4 ". But of course, all the results of our study could be obtained in the traditional notation of gravitomagnetism with the relation $\boldsymbol{k}=\frac{\boldsymbol{B}_{g}}{4}$.

\subsection{From Linearized General Relativity to Gravitational Redshift}

In our approximation, we are going to seek for a relation of the gravitational potential that contain the gravitic component in addition to the gravity's term. It could be seen as a correction of the traditional gravitational potential that take in account only the gravity field. By this way (in the linearized approximation), others relations in which the gravitational potential intervenes could be adapted to take in account the gravitic field.

From the previous relation (16), one can write:

$$
\begin{gathered}
\mathrm{d} s^{2}=\left(1+\frac{2 \varphi}{c^{2}}-\frac{8 \boldsymbol{H} \cdot \mathrm{d} \boldsymbol{x}}{c^{2} \mathrm{~d} t}\right) c^{2} \mathrm{~d} t^{2}-\left(1-\frac{2 \varphi}{c^{2}}\right) \sum\left(\mathrm{d} x^{i}\right)^{2} \\
\mathrm{~d} s^{2}=\left(1+\frac{2(\varphi-4 \boldsymbol{H} \cdot(\mathrm{d} \boldsymbol{x} / \mathrm{d} t))}{c^{2}}\right) c^{2} \mathrm{~d} t^{2}-\left(1-\frac{2 \varphi}{c^{2}}\right) \sum\left(\mathrm{d} x^{i}\right)^{2}
\end{gathered}
$$

One can then define, in this approximation, a gravitational potential that could be qualified as "corrected" in the sense that the gravitic term is added to the traditional gravitational potential as a corrected term (with the velocity of the test particle, $\boldsymbol{v}=\mathrm{d} \boldsymbol{x} / \mathrm{d} t)$ :

$$
\varphi_{C O R R}=\varphi-4 \boldsymbol{H} \cdot \boldsymbol{v}=\varphi+\varphi_{K 1}
$$

We can then apply these relations for the gravitational redshift:

$$
1+z=\left(\frac{1-\frac{2}{c^{2}} \varphi_{C O R R, E}}{1-\frac{2}{c^{2}} \varphi_{C O R R, R}}\right)^{1 / 2} \sim\left(\frac{1-\frac{2}{c^{2}}\left(\varphi_{E}+\varphi_{K 1, E}\right)}{1-\frac{2}{c^{2}}\left(\varphi_{R}+\varphi_{K 1, R}\right)}\right)^{1 / 2}
$$




\section{Gravitic Field and Gravitational Redshift at the Center of Our Galaxy}

\subsection{Simplified Relations for the Gravitational Redshift of SO-2}

As demonstrated in [2], in the case of SO-2, the redshift due to general relativity, $z_{G R}$, can be obtained with the following term (due on one hand to the gravity field of $\operatorname{SgrA}^{*}, \varphi_{\mathrm{SgrA}^{*}}$ and on the other hand to the gravitic field of $\operatorname{Sgr} A^{*}$, $\left.\varphi_{K 1-\mathrm{SgrA}}\right)$ :

$$
\begin{aligned}
& z_{G R} \sim-\frac{1}{c^{2}} \varphi_{\mathrm{SgrA}^{*}}-\frac{1}{c^{2}} \varphi_{K 1-\mathrm{SgrA}^{*}}=z_{G}+z_{H} \\
& \varphi_{\mathrm{SgrA}^{*}}=-\frac{G M_{\mathrm{SgrA}^{*}}}{r_{\mathrm{SO}-2 \leftrightarrow \mathrm{SgrA}^{*}}} \\
& \varphi_{K 1-\mathrm{SgrA}^{*}}=-\frac{4 K_{1} v}{r_{\mathrm{SO}-2 \leftrightarrow \mathrm{SgrA}^{*}}}
\end{aligned}
$$

with $K_{1}$ the factor of the gravitic field in the punctual definition (from Poisson Equation (9) the gravitic field can be written $k \propto K_{1} / r^{2}$ and in this punctual approximation $\left.\|\boldsymbol{H}\| \sim \frac{K_{1}}{r}\right)[3]$.

The two components of redshift of SO-2 due to general relativity is then:

$$
\begin{aligned}
& z_{G} \sim \frac{G M_{\mathrm{SgrA}^{*}}}{r_{\mathrm{SO}-2 \leftrightarrow \mathrm{SgrA}^{*}} c^{2}} \\
& z_{H} \sim \frac{4 K_{1} v}{r_{\mathrm{SO}-2 \leftrightarrow \mathrm{SgrA}^{*}} c^{2}}
\end{aligned}
$$

Because the term $z_{H}$ depends on the velocity of SO-2 $(v)$, the effect of this term can be detectable only at the periastron at which the velocity is large enough.

Let's remind that there is also another component for the computation of the redshift due to special relativity $z_{S R} \sim z_{G}$.

\subsection{Theoretical Values for the Gravitational Redshift of SO-2}

In the paper explaining the dark matter [3], the gravitic field of the studied galaxies (more specifically the factor $K_{1}$ for the whole galaxy, $K_{1 \_\mathrm{GAL}}$ and not for the only SMBH of the center of the galaxy) is expected to be in the following interval:

$$
10^{24} \leq K_{1 \_\mathrm{GAL}} \leq 10^{24.9}
$$

If we adapt these values of whole galaxy, $K_{1 \_\mathrm{GAL}}$ (for a typical mass of $\left.2 \times 10^{42} \mathrm{~kg}\right)$ to SMBH of the center of galaxy, $K_{1}\left(M_{\mathrm{SgrA}^{*}} \sim 4 \times 10^{6} \times 2 \times 10^{30} \mathrm{~kg}\right)$ [7] [8] by applying a factor of $M_{\mathrm{SgrA}^{*}} / M_{\mathrm{GAL}} \sim 8 \times 10^{36} /\left(2 \times 10^{42}\right)=4 \times 10^{-6}$, (23) becomes:

$$
10^{18.6} \leq K_{1} \leq 10^{19.5}
$$

with the distance of SO-2 to $\operatorname{SgrA}^{*}\left(r_{\mathrm{SO}-2 \leftrightarrow \mathrm{SgrA}^{*}} \sim 2 \times 10^{13} \mathrm{~m}\right)$ and the velocity at the periastron of SO-2 $v \sim 8 \times 10^{6} \mathrm{~m} \cdot \mathrm{s}^{-1}$ [7] [8], (22) gives: 


$$
z_{H} \sim \frac{4 K_{1} 8 \times 10^{6}}{2 \times 10^{13} \times 9 \times 10^{16}} \sim 2 K_{1} \times 10^{-23}
$$

And with the previous values of $K_{1}$ (24), one obtains:

$$
\begin{aligned}
& 10^{-4.1} \leq z_{H} \leq 10^{-3.2} \\
& 8 \times 10^{-5} \leq z_{H} \leq 6 \times 10^{-4}
\end{aligned}
$$

If we compare to $z_{G},(22)$ gives:

$$
z_{G} \sim \frac{6 \times 10^{-11} \times 8 \times 10^{36}}{2 \times 10^{13} \times 9 \times 10^{16}} \sim 3 \times 10^{-4}
$$

Let's remind that the expected redshift (expected for the main part of the researchers) is only $z=z_{G}+z_{S R}$ with $z_{S R} \sim z_{G}$, it would then give for the redshift of SO-2:

$$
z=z_{G}+z_{S R} \sim 6 \times 10^{-4}
$$

The added term that I predict is then at least close to the same order of magnitude of each term $\left(z_{S R}\right.$ or $\left.z_{G}\right)$.

Translated in term of velocity, these redshifts $\left(v_{z_{H}}\right.$ for the sample of the studied galaxies) are:

$$
\begin{aligned}
& 30 \mathrm{~km} \cdot \mathrm{s}^{-1} \leq v_{z_{H}} \leq 180 \mathrm{~km} \cdot \mathrm{s}^{-1} \\
& v_{z_{S R}} \sim 100 \mathrm{~km} \cdot \mathrm{s}^{-1} \\
& v_{z_{G}} \sim 100 \mathrm{~km} \cdot \mathrm{s}^{-1}
\end{aligned}
$$

Instead of the expected value $v_{z} \sim 200 \mathrm{~km} \cdot \mathrm{s}^{-1}$, one then should have:

$$
v_{z} \geq 230 \mathrm{~km} \cdot \mathrm{s}^{-1}
$$

\subsection{Observed Values for the Gravitational Redshift of SO-2}

If we look at the recent observations (Figure 1) on the redshift of SO-2 [1], we first remark that general relativity is once again confirmed as the more accurate theory of gravitation.

But we also detect a discrepancy, only at the periastron. Instead of $v_{z} \sim 200 \mathrm{~km} \cdot \mathrm{s}^{-1}$, the measure gives $v_{z} \sim 240 \mathrm{~km} \cdot \mathrm{s}^{-1}$. The value matches well with the gravitic field (30) expected in the frame of the explanation of the dark matter [3].

We can specify that the effect of the gravitic field of SgrA* on SO-2 was expected to be negligible all around the orbit of SO-2. Only at the periastron, a discrepancy was expected. The value of this discrepancy was expected to be at least $v_{z_{H}} \geq 30 \mathrm{~km} \cdot \mathrm{s}^{-1}$. The observation of [1] confirms these three characteristics.

\section{Conclusions}

With the passage of SO-2 star at its closest position to SgrA*, the gravitational theories can be tested. Once again, General Relativity is the best gravitational theory to explain these observations. But these measures on the redshift of SO-2 


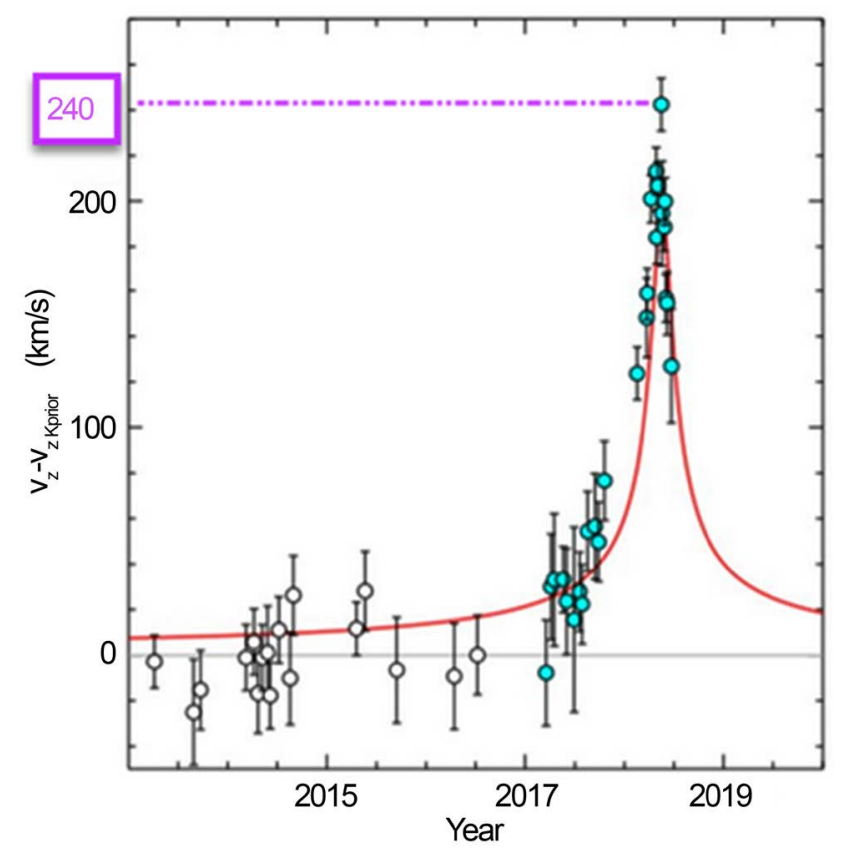

Figure 1. Residual velocity for the best fitting prior Keplerian $\mathrm{K}_{\text {prior }}(f=0$, grey) and the same orbit with $f=1$ (red $\mathrm{GR}_{\text {prior }}$ ). $\mathrm{K}_{\text {prior }}$ was constructed from all 1992-2018 astrometric data with NACO \& GRAVITY and the SINFONI data between 2004 and 2016 (open black circles). The 2017/2018 SINFONI data points (black circles with cyan shading) can then be added to test if the spectroscopic data around pericentre follow $\mathrm{K}_{\text {prior }}$ or the $\mathrm{GR}_{\text {prior }}$ predicted from $\mathrm{K}_{\text {prior }}$ (Figure adapted with permission from [1]. Copyrighted by A \& A).

reveal a discrepancy at its periastron. It doesn't mean that general relativity failed. Indeed, in a previous paper [2], it was predicted that general relativity would imply such a discrepancy. This discrepancy was expected in the frame of an explanation of dark matter [3] compliant with genral relativity (and without exotic matter). The gravitic field (second component of general relativity similar to magnetic field) of the large astrophysical structures (galaxies, clusters...) would be greater than expected. In this frame of dark matter explanation, the effect of the gravitic field of SgrA* on SO-2 was expected to be negligible all around the orbit of SO-2. Only at the periastron, a discrepancy was expected. The value of this discrepancy was expected to be at least $v_{z_{H}} \geq 30 \mathrm{~km} \cdot \mathrm{s}^{-1}$. The observation confirms these three characteristics. This discrepancy would be then not only a confirmation of the general relativity but also a confirmation that the gravitic field of large astrophysical structures is systematically underestimated. It then leads to an important positive clue for the explanation of dark matter as a gravitic field of clusters.

We can remind that others success of this solution has been obtained. This solution predicted necessarily the movement of dwarf satellites galaxies along planes [3] [9]. Several observations confirm it [10] [11]. Some observations leaded to several experimental relations [12]. These relations can be obtained with this solution [13]. To end, one can note that the capacity of this solution to solve so disparate problems is remarkable. 


\section{Conflicts of Interest}

The author declares no conflicts of interest regarding the publication of this paper.

\section{References}

[1] Abuter, R., et al. (2018) Detection of the Gravitational Redshift in the Orbit of the Star S2 near the Galactic Centre Massive Black Hole. arXiv:1807.09409.

[2] Le Corre, S. (2018) Gravitational Redshift and Dark Matter: Application to SO-2's Case. OALib, 5, 1-10.

[3] Le Corre, S. (2015) Dark Matter, a New Proof of the Predictive Power of General Relativity. https://arxiv.org/abs/1503.07440

[4] Adler, R.J. (2015) The Three-Fold Theoretical Basis of the Gravity Probe B Gyro Precession Calculation. https://arxiv.org/abs/1405.5511

[5] Hobson, M., et al. (2006) General Relativity. Cambridge University Press, Cambridge. https://doi.org/10.1017/CBO9780511790904

[6] Mashhoon, B. (2008) Gravitoelectromagnetism: A Brief Review. https://arxiv.org/abs/gr-qc/0311030

[7] Schödel, R., et al. (2002) A Star in a 15.2-Year Orbit around the Supermassive Black Hole at the Centre of the Milky Way. https://www.nature.com/articles/nature01121

[8] Gillessen, S., et al. (2017) An Update on Monitoring Stellar Orbits in the Galactic Center. arXiv:1611.09144.

[9] Le Corre, S. (2015) Dark Matter and Planes of Corotating Satellite Galaxies. https://hal.archives-ouvertes.fr/hal-01239270

[10] Phillips J.I., Cooper, M.C., Bullock, J.S. and Boylan-Kolchin, M. (2015) Are Rotating Planes of Satellite Galaxies Ubiquitous? Monthly Notices of the Royal Astronomical Society, 453, 3839-3847. https://doi.org/10.1093/mnras/stv1770

[11] Müller, O., Pawlowski, M.S., Jerjen, H. and Lelli, F. (2018) A Whirling Plane of Satellite Galaxies around Centaurus A Challenges Cold Dark Matter Cosmology. Science, 359, 534-537. https://doi.org/10.1126/science.aao1858

[12] McGaugh, S.S., Lelli, F. and Schombert, J.M. (2016) The Radial Acceleration Relation in Rotationally Supported Galaxies. Physical Review D, 117, Article ID: 201101. https://arxiv.org/abs/1609.05917

[13] Le Corre, S. (2017) Dark Matter without New Matter Is Compliant with General Relativity. Open Access Library Journal, 4, 1-12. https://doi.org/10.4236/oalib.1103877 